\title{
Regionale Anpassung an den Klimawandel - Ein Überblick mit Empfehlungen für Kommunen in Baden-Württemberg
}

\author{
Simone Häußler ${ }^{1} \mathbb{D}$ - Martina Hofmann ${ }^{1} \cdot$ Martin Müller $^{2}$ \\ Online publiziert: 3. Juli 2020 \\ (c) Der/die Autor(en) 2020
}

\section{Zusammenfassung}

Der Klimawandel wird sich in Baden-Württemberg im Wesentlichen mit einer Zunahme an Extremwetterlagen von Hitze und Starkregen auswirken. Die Kommunen stehen vor der Herausforderung, mit den bereits eingetretenen und den zu erwartenden klimatischen Veränderungen angemessen umzugehen. In Deutschland gibt es auf Bundesebene seit 2006 erkennbare Bestrebungen, das Thema der Anpassung an den Klimawandel zu institutionalisieren. Es sind allerdings primär die Länder, die spezifische Programme für ihren jeweiligen geografischen Kontext initiieren und umsetzen müssen. Den kommunalen Entscheidern kommt hierbei eine zentrale Verantwortung zu, denn sie sind auf lokaler Ebene gefordert, den Herausforderungen des Klimawandels zu begegnen. Hemmende Faktoren für kommunale Anpassungsstrategien sind dabei u.a. die fehlende Benennung von klaren Zuständigkeiten und die weitgehende Unverbindlichkeit von Richtlinien und Beschlüssen. An diesen Punkten sollte angeknüpft werden. Die strategische Einbindung von regionalen Stakeholdern könnte dazu beitragen, die überregionale Anpassungsplanung wirksam in die Kommunalplanung zu überführen.

Schlüsselwörter Geographie $\cdot$ Wetterextreme $\cdot$ Klimaanpassung $\cdot$ Regionale Stakeholder $\cdot$ Verantwortlichkeiten

\begin{abstract}
Climate change will have a major impact in Baden-Württemberg in the form of an increase in extreme weather conditions of heat and heavy rain. The local communities are faced with the challenge of dealing properly with the climatic changes that have already occurred and those that are expected. In Germany, there have been evident efforts at a federal level since 2006 to establish the issue of adaptation to climate change on an institutional level. It is primarily the federal states that must initiate and implement specific programmes for their respective geographical contexts. The municipal decision-makers have a central responsibility in this regard, as they are expected to meet the challenges of climate change at a local level. Factors that inhibit municipal adaptation strategies are, among others, the lack of clear responsibilities and the fact that the directives and resolutions are largely non-binding. These issues should be followed up. The strategic integration of regional stakeholders could contribute to the effective transfer of transregional adaptation planning into municipal planning.
\end{abstract}

Keywords Geography $\cdot$ Extreme Weather Conditions $\cdot$ Climate adaptation $\cdot$ Regional Stakeholders $\cdot$ Responsibilities

\section{Einleitung}

Prognosen zufolge (LUBW 2019) werden in verschiedenen Regionen Baden-Württembergs verstärkt Hitze- und Stark-

Simone Häußler, M. Sc.

Simone.Haeussler@hs-aalen.de

1 Elektrotechnik - Erneuerbare Energien, Hochschule Aalen Technik und Wirtschaft, Anton-Huber-Str. 25, 73430 Aalen, Deutschland

2 Institut für Nachhaltige Unternehmensführung, Fakultät für Mathematik und Wirtschaftswissenschaften, Universität Ulm, Helmholtzstr. 18, 89081 Ulm, Deutschland regenereignisse erwartet. Vor dem Hintergrund der Relevanz von Wetterextremen sind die präventiven Maßnahmen auf kommunaler Ebene oftmals noch nicht weit genug entwickelt. Vor allem in mittelgroßen und kleinen Kommunen (gemäß der Eingruppierung des Bundesinstituts für Bau-, Stadt-, und Raumforschung) werden Lücken sichtbar (Vetter et al. 2017, S. 328). Häufig sind weder verantwortliche Akteure benannt noch verbindliche Ziele verankert (Koerth 
et al. 2019). Gleichzeitig sind es jedoch die Kommunen, die von Klimafolgen auf lokaler Ebene betroffen sein werden.

Dieser Artikel hat das Ziel, einen aktuellen Überblick zum Thema der regionalen Klimaanpassung zu geben. Die Darstellung der spezifischen Geografie Baden-Württembergs und die Prognose der zukünftigen klimatischen Entwicklungen zeigen einen akuten Handlungsbedarf auf. Auf Basis einer systematischen Literaturrecherche (SLR) der Anpassungsstrategien auf Bundes- und auf Länderebene in Deutschland werden exemplarisch am Beispiel Baden-Württemberg die Rahmenbedingungen für kommunale Aktivitäten dargestellt. Aus den Ergebnissen der intensiven Auseinandersetzung der Autor*innen mit regionaler Anpassungsstrategie werden die akuten Hemmnisse auf kommunaler Ebene aufgezeigt und Handlungsempfehlungen abgeleitet.

\section{Anpassung an den Klimawandel auf Bundesebene}

Innerhalb der EU-Kommission verabschiedete die DG CLIMA (Generaldirektion Climate Action) 2013 erstmals eine europäische Strategie zur Anpassung an den Klimawandel. Deren Zielsetzung besteht primär darin, die Resilienz der europäischen Länder gegenüber Klimaveränderungen zu stärken (Marx 2017, S. 10f.). Auf Bundesebene arbeiten die Ministerien auf freiwilliger, konsensualer Basis, gemäß eines network mode of governance für Anpassung an den Klimawandel (Vetter et al. 2017, S. 327) zusammen. Im Oktober 2006 wurde das Kompetenzzentrum für Klimafolgen und Anpassung (KomPass) als zentrale Stelle für Klimafolgenanpassung im Umweltbundesamt gegründet. Dessen Aufgaben bestehen im Wesentlichen in der Vermittlung wissenschaftlicher Grundlagen sowie in der interinstitutionellen und thematischen Vernetzung der Länder (Haße und Daschkeit 2012). Die nationalen Adaptionspläne wurden im Dezember 2008 durch die Bundesregierung mit der Deutschen Anpassungsstrategie an den Klimawandel (DAS) formuliert. Diese initiierte erstmals einen politischen Rahmen für die Klimaanpassung in Deutschland und zeigt mögliche nationale Auswirkungen des Klimawandels auf (Vetter und Schauser 2013). Nach dem offiziellen Inkrafttreten der DAS 2008 wurde der bis dato informelle Arbeitskreis zur Anpassung zu einer beratenden und ressortübergreifenden „Interministeriellen Arbeitsgruppe Anpassungsstrategie“" (IMA Anpassungsstrategie). Die Mitglieder der IMA Anpassungsstrategie sind zusammen mit dem 2009 gegründeten „Ständigen Ausschuss zur Anpassung an die Folgen des Klimawandels" (StA AFK) für die Weiterentwicklung der DAS und für die Abstimmung und Koordination der Aktionen und Maßnahmen zur Klimaanpassung in Bund und Ländern verantwortlich. Mit der Weiterentwicklung der
DAS im Jahr 2011, dem „Aktionsplan Anpassung der Deutschen Anpassungsstrategie an den Klimawandel" (APA), wurden die bisher beschriebenen Handlungsfelder (Wasser, Infrastruktur, Raumplanung und Bevölkerungsschutz, Land, Gesundheit, Wirtschaft) durch konkrete Umsetzungsempfehlungen und Aktivitäten ergänzt. Die Verstetigung der DAS soll zudem durch den regelmäßigen Fortschrittsbericht mit spezifischen Aktionsplänen gewährleistet werden. Der Ende 2015 erschienene Fortschrittsbericht basiert inhaltlich auf dem ebenfalls 2015 erschienenen Monitoringbericht zur Deutschen Anpassungsstrategie. Der erste Fortschrittsbericht beinhaltet außerdem den Aktionsplan Anpassung (APA II) sowie die Ergebnisse der deutschen Vulnerabilitätsanalyse. Dem fortlaufenden Monitoring (gemäß 2. Monitoringbericht 2019), ist zudem die aktualisierte Darstellung einer Reihe deutschlandweiter Erhebungen von Klimaauswirkungen anhand statistischer Zeitreihen zu entnehmen (UBA 2019).

Die Evaluierung der Fortschritte in der politischen Institutionalisierung von Klimaanpassung wird mit der „Politikanalyse zur Evaluation der DAS“ 2019 vorgenommen. Diese fokussiert sich im Gegensatz zum Monitoringbericht insbesondere auf die Wirkungsanalyse und die Weiterentwicklung der DAS (Gaus et al. 2019, S. 12).

Für das Jahr 2020 ist die Publikation des 2. Fortschrittsberichts zur DAS (einschließlich des aktualisierten Aktionsplans Anpassung der Deutschen Anpassungsstrategie an den Klimawandel APA II) vorgesehen. Gemäß dem Auftrag der Bundesregierung im Fortschrittsbericht 2015 hat das Umweltbundesamt die neue Vulnerabilitätsanalyse in Auftrag gegeben. Diese soll im Jahr 2021 veröffentlicht werden und ein aktualisiertes Gesamtbild nach neuesten wissenschaftlichen Erkenntnissen vermitteln (Tab. 1).

Ein direkter Bezug zu den Empfehlungen der Bundesregierung zur Klimaanpassung findet sich in den Vorschriften

Tab. 1 Veröffentlichungen zum Thema Anpassung an den Klimawandel auf Bundesebene. (Quelle: eigene Darstellung)

\begin{tabular}{ll}
\hline Jahr & Name der Veröffentlichung \\
\hline 2008 & $\begin{array}{l}\text { Deutsche Anpassungsstrategie an den Klimawandel } \\
\text { (DAS) }\end{array}$ \\
2011 & $\begin{array}{l}\text { Aktionsplan Anpassung der Deutschen Anpassungs- } \\
\text { strategie } \\
\text { an den Klimawandel (APA) }\end{array}$ \\
& $\begin{array}{l}\text { 1. Fortschrittsbericht zur DAS einschließlich } \\
2015\end{array}$ \\
& - Deutsche Vulnerabilitätsanalyse \\
2015 und & Monitoringberichte zur Deutschen Anpassungsstrategie \\
2019 & Politikanalyse zur Evaluation der DAS \\
2019 & 2. Fortschrittsbericht zur DAS \\
Geplant: & (einschließlich APA III) \\
2020 & 2. Klimawirkungs- und Vulnerabilititätsbericht \\
Geplant: & \\
2021 &
\end{tabular}


des Raumordnungsgesetzes (ROG), des Baugesetzbuches (BauGB), des Wasserhaushaltsgesetzes (WHG) (Bubecka et al. 2016) sowie im Gesetz über die Umweltverträglichkeitsprüfung (UVPG).

\section{Klimaanpassung als Strategie in Baden- Württemberg}

Die Landesregierung von Baden-Württemberg sieht es als eine ihrer zentralen Aufgaben, an der Bewältigung der Herausforderungen des Klimawandels mitzuwirken. Im Juli 2013 wurde das „Gesetz zur Förderung des Klimaschutzes in Baden-Württemberg" (nachfolgend KSG) im Landtag von Baden-Württemberg verabschiedet. Dessen Zweck besteht darin, einen angemessenen Beitrag zum Klimaschutz zu leisten und gleichzeitig zur Klimaanpassung (gemäß § 4 KSG) beizutragen. Mit dem KSG werden die konkreten Zielvorgaben der Landesregierung für die stufenweise Reduzierung von Treibhausgasemissionen bis zum Jahr 2020 um $25 \%$ gegenüber 1990 und um $90 \%$ bis zum Jahr 2050 präzisiert (UMBW 2015). Zudem ist in den Zielen des KSG die Verpflichtung zur Entwicklung einer Klimaanpassungsstrategie verankert, um mit den Konsequenzen des Klimawandels in Baden-Württemberg umzugehen. Zur Erreichung der im KSG formulierten Klimaschutzziele hat die Landesregierung die Initiierung eines integrierten Energieund Klimaschutzkonzepts (nachfolgend IEKK) angestoBen. Dieses soll anhand konkreter Strategien zu sichtbaren Fortschritten im Klimaschutz führen. Die im IEKK vorrangig betrachteten Handlungsbereiche sind Stromversorgung, Wärmebedarf, Verkehr, Land- und Forstwirtschaft, Landnutzung und Stoffströme (UMBW 2014). Die Besonderheit des IEKK gegenüber vergleichbaren Landes-Klimaschutzkonzepten besteht in der Beteiligungsmöglichkeit Dritter. Eine Rechtsverbindlichkeit zur Einhaltung der im IEKK formulierten Maßnahmen für die Kommunen im Land, vergleichbar mit dem Klimaschutzkonzept des Landes Rheinland-Pfalz (LKSG RP), besteht nicht (Flaskuehler 2018, S. 272). Beide Konzepte (IEKK und LKSG RP) bieten den jeweiligen kommunalen Akteuren ihres Landes eine „Entscheidungshilfe“. Aktualisiert wird das IEKK in regelmäßigen Zeiträumen von 5 Jahren auf Basis eines Monitoringberichts. Dieses Monitoring enthält neben dem IEKKStatusbericht auch die Fortschreibungen der allgemeinen Ziele des KSG und die Einbindung in die Klimaschutzmaßnahmen des Bundes und der Europäischen Union. Auf Basis des IEKK wurde 2015 die „Anpassungsstrategie Baden-Württemberg" veröffentlicht. Diese soll auf die zu erwartenden Auswirkungen des Klimawandels vorbereiten, um die Vulnerabilität des Landes zu mindern, Klimafolgekosten zu senken und Handlungsoptionen aufzuzeigen. Um die Klimaschutz- und Klimaanpassungsbestrebungen des Landes voranzubringen, wurde zudem 2016 die Fortschreibung mit dem Koalitionsvertrag beschlossen. Acht Eckpunkte zur Weiterentwicklung des Klimaschutzgesetzes sollen darin zukünftig umgesetzt werden. Aktuell wird ein Gesetzentwurf zur Änderung des Klimaschutzgesetzes erarbeitet (UMBW 2019).

\section{Bisherige und zukünftige klimatische Entwicklungen am Beispiel Baden- Württemberg}

Aufgrund der geografischen Heterogenität dieses Bundeslandes variieren die bisherigen und die zu erwartenden klimatischen Entwicklungen auf regionaler Ebene (Deutschländer und Mächel 2017, S. 51). Klimaszenarien prognostizieren näherungsweise Entwicklungen der Umwelt und bilden die Randbedingungen für Klimamodellierungen (Ranke 2019, S. 75). Mögliche Entwicklungen und deren Auswirkungen werden nachfolgend exemplarisch thematisiert.

Seit der Jahrtausendwende zeichnet sich der Trend eines kontinuierlich steigenden Temperaturniveaus in BadenWürttemberg ab. Zwischen den Jahren 2000 und 2020 wurden die höchsten Jahresdurchschnittstemperaturen seit dem Beginn der Wetteraufzeichnungen im Jahr 1881 ermittelt. Auf Basis von Auswertungen regionaler Messungen wurden Projektionen zur möglichen Klimaentwicklung in Baden-Württemberg erstellt. Als Kennwerte der Betrachtung dienten die Jahresmitteltemperatur (in Grad Celsius $\left[{ }^{\circ} \mathrm{C}\right]$ ), die Sommertage (Tagesmaximaltemperatur $\geq 25^{\circ} \mathrm{C}$ ) und die heißen Tage (Tagesmaximaltemperatur $\geq 30^{\circ} \mathrm{C}$ ). Tendenziell werden sich die bisherigen Trends (Hitzesommer, Starkniederschläge im Winter), welche seit der Jahrtausendwende zu verzeichnen sind, landesweit intensivieren (LUBW 2019). Für die Durchschnittstemperatur in Baden-Württemberg werden weiterhin Zunahmen für die Sommer- und Wintermonate erwartet. Die höheren Lagen der Mittelgebirge werden weniger betroffen sein als die Tieflandregionen Baden-Württembergs (UMBW 2015). Die Anzahl der heißen Tage hat sich seit dem Betrachtungszeitraum 1961-1990 von 5 Tagen auf 21 Tage (2018) im Mittel vervierfacht (LUBW 2019; siehe Abb. 1). Während der europaweiten Hitzewelle im Jahr 2003 wurden aufgrund einer ausgeprägten Omegalage zwischen April und September Temperaturrekorde erreicht. Der Südwesten gehörte mit 27 heißen Tagen zu den Gebieten mit den höchsten Tagesmittelwerten Deutschlands (Schindler und Mayer 2007). Besonders von Hitze betroffen sind der Oberrheingraben, das Neckarbecken und das Bodenseegebiet (UMBW 2015).

Mit 17 heißen Tagen und einer Jahresmitteltemperatur von $9,9^{\circ} \mathrm{C}$ gehört das Jahr 2019 zu den 4 heißesten Jahren in Baden-Württemberg (LUBW 2020). Gleichzeitig war Baden-Württemberg im Jahr 2019 mit mehr als 2501/m² 
Abb. 1 Anzahl heißer Tage in Baden-Württemberg für die Jahre 2003 und 2018 im Vergleich zum Zeitraum 1961-1990. (Quelle: LUBW 2019; berechnet nach Daten des DWD)
$1961-1990$
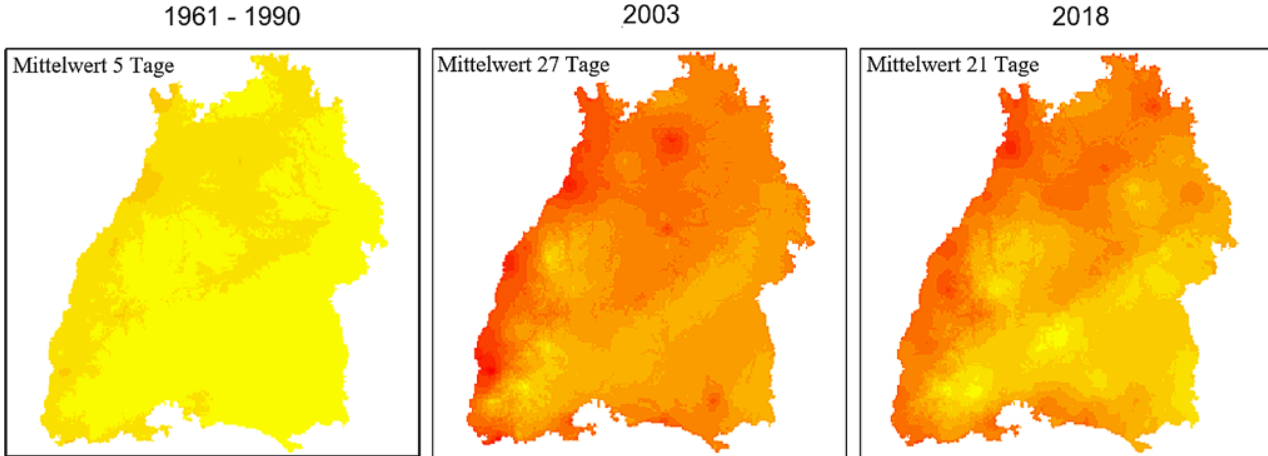

Anzahl heißer Tage in Baden-Württemberg, definiert durch ein Temperaturmaximum $\geq 30$ Grad Celsius.

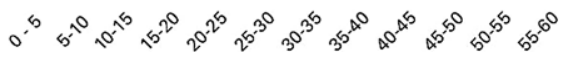

auch das niederschlagsreichste Bundesland Deutschlands. Die anhaltende Hitze der Sommermonate führte jedoch zu sehr hohen Verdunstungsraten, wodurch die Böden nicht nachhaltig profitieren konnten (DWD 2019). Zudem konzentrierte sich die Jahresgesamtregenmenge auf die Wintermonate, was eine Zunahme lokaler Starkniederschläge zur Folge hatte. Klimatische Projektionen der monatlichen Wasserbilanz deuten auf eine Erhöhung zwischen Januar und Juni sowie zwischen September und Dezember für die nahe Zukunft hin. Für die Sommermonate (Juli bis August) wird eine Verringerung der Niederschläge erwartet (UMBW 2015).

Der im März 2020 erschienene Bericht der Landesanstalt für Umwelt zur klimatischen Einordnung des Vorjahres Wieder außergewöhnlich warm und heiß, mit Nachwirkungen des Trockenjahrs 2018 bestätigt den anhaltenden Trend zu hohen Temperaturwerten und Trockenperioden (LUBW 2020).

Abb. 2 Schäden durch das Starkregenereignis 2016 in Schwäbisch Gmünd. (Quelle: Stadt Schwäbisch Gmünd)

\section{Regionale Auswirkungen des Klimawandels in Baden-Württemberg}

Baden-Württemberg wird den Klimaprognosen zufolge im Bundesvergleich eine niederschlagsreiche Region bleiben. Die Änderung der Niederschlagsmuster kann jedoch nachhaltige Auswirkungen auf Sektoren, wie z.B. Infrastruktur und Bauwirtschaft, Energieversorgung, Wasserwirtschaft und Landwirtschaft, mit sich bringen. Lokale Starkregenereignisse, die zum Teil zu enormen Schäden an landwirtschaftlichen Kulturen führten, sowie dürre, trockene Sommer wie im Jahr 2018 könnten sich als Folge der Niederschlagsverlagerung häufen (LUBW 2019). Extremwetterlagen wie das Hochwasser von 2013 (betreffend u. a. die Kreise Reutlingen, Sigmaringen, Tübingen und Zollernalbkreis) oder der Starkregen im Frühsommer 2016 (betreffend u.a. die Schwäbische Alb, s. Abb. 2) führten in der Vergangenheit bereits zu enormen Sachschäden und Schäden an der Natur.

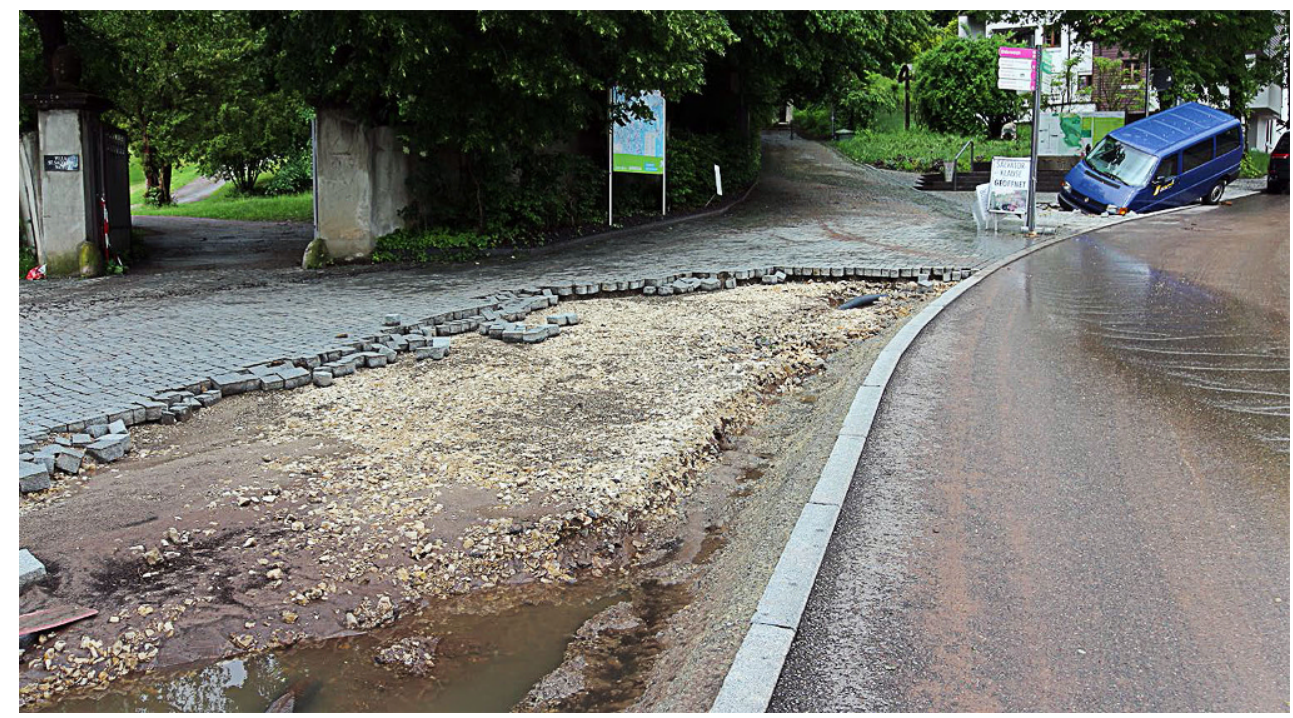


Gemäß aktueller Klimaprojektionen des Forschungsprojekts IMK-TRO werden sich zudem die maximalen Windgeschwindigkeiten und deren Frequenz erhöhen. Als besonders vulnerabel gegenüber Stürmen wurden Kommunen mit einer hohen Industriedichte wie Esslingen am Neckar, Pforzheim, Reutlingen, Heilbronn, Ludwigsburg und Göppingen eingestuft (Fath et al. 2014, S. 50). Die prognostizierte Temperaturentwicklung der Sommermonate wird zu vermehrten heißen Tagen in Baden-Württemberg führen. Diese Temperaturextreme begünstigen (z.B. durch einen Mangel an Frischluftschneisen, Grünflächen und Verschattung) die Entstehung von lokalen Hitzespots. Aufgrund ihrer geografischen Besonderheiten werden Städte wie Stuttgart (Klimaks 2012) oder Karlsruhe als besonders gefährdet eingestuft.

Für gegenüber Hitze sensiblen Gruppen stellen erhöhte Temperaturen ein gesundheitliches Risiko dar (Beckmann und Hiete 2020). Städte und Gemeinden in Baden-Württemberg müssen sich zukünftig stärker auf den Hitzeschutz in Städten, z. B. durch gezielte Maßnahmen und Informationsbereitstellung, einstellen.

\section{Kommunale Klimaanpassungsstrategien in Baden-Württemberg}

Die formalen Voraussetzungen für die Integration von Anpassung in kommunale Planungen wurden bereits erfolgreich geschaffen (Bubecka et al. 2016). Klimaschutz und Anpassung an den Klimawandel sind bereits seit der Baunovelle 2006 ein fester Bestandteil des städtebaulichen Rechts. Entsprechende Anpassungen in den kommunalen Planungsund Entscheidungsprozessen (z. B. durch den kontinuierlichen Einbezug von beratenden Stellen und die Benennung von Verantwortlichen) werden bislang jedoch nur vereinzelt und nicht systematisch vorgenommen (vgl. Darstellung in Abb. 3). In der Praxis ist die Thematik der Klimaanpassung vor allem in mittelgroßen und kleinen Städten und Gemeinden noch nicht mehrheitlich strukturell institutionalisiert (Vetter et al. 2017, S. 331f.).

Über eigene Klimaanpassungskonzepte verfügen die Städte Stuttgart (Klimaks), Ludwigsburg (Klik) und Karlsruhe (Anpassung an den Klimawandel - Bestandsaufnahme und Strategie für die Stadt Karlsruhe). Die Anpassungsstrategie der Landeshauptstadt umfasst einen gewichteten Katalog von 53 Maßnahmen aus allen Anpassungsbereichen analog der Deutschen Anpassungsstrategie. Berücksichtigt werden neben planerischen (Entsiegelung, Begrünung) und baulichen Aspekten (Sonnenschutz, Limitierung Fensterflächen) auch Aspekte der Öffentlichkeitsarbeit (Klimaks 2012). Das strategische Anpassungskonzept der Stadt Ludwigsburg setzt mit 22 formulierten Maßnahmen einen starken Fokus auf Hitzeschutz (z.B. durch die systematische Begrünung von Dächern, Plätzen und Mauern). Die am stärksten von Hitze betroffenen Stadtstrukturen wurden lokalisiert und in einem Gesamtplan mit den korrespondierenden Maßnahmen dargestellt. Die Stadt Karlsruhe gehört aufgrund ihrer Lage am Oberrheingraben zu den deutschen Kommunen mit den höchsten gemessenen Durchschnittstemperaturen. Auch hier werden erhöhte Auftrittswahrscheinlichkeiten für Hitzetage in der nahen Zukunft prognostiziert, weshalb das Thema Anpassung in der Stadtplanung verstärkt im Konzept fixiert wird. Eine Weiterentwicklung der Karlsruher Anpassungsstrategie, insbesondere in Bezug auf Hitzespots (sogenannte Urban-Heat-Phänomene), findet sich im Städtebaulichen Rahmenplan Klimaanpassung 2015 (Obert 2017, S. 500f.).

Als Gemeinsamkeit der kommunalen Anpassungsstrategien der 3 betrachteten Städte wurden zunächst No-Regret-Maßnahmen identifiziert. Bei diesen Maßnahmen übersteigt der unter bereits bestehenden Klimabedingungen erzielte Nutzen die anfallenden Kosten. No-Regret-Maßnahmen (wie beispielsweise Stadtgrün) gelten ökologisch, sozial und wirtschaftlich grundsätzlich als sinnvoll oder vorteilhaft (z.B. Koerth et al. 2019). Die Städte, die bereits über ein eigenes Klimaanpassungskonzept verfügen, konnten dies zudem an bereits geleistete Vorarbeiten, wie z.B. Energie- oder Klimaschutzkonzepte, anknüpfen.

Neben den genannten Vollkonzepten gibt es auch projektbasierte Anpassungsmaßnahmen. Exemplarisch werden nachfolgend Angebote zur Unterstützung von Kommunen aufgezeigt.

- Klimalotse

Das Förderprogramm Klimopass des Ministeriums für Umwelt, Klima und Energiewirtschaft Baden-Württem-
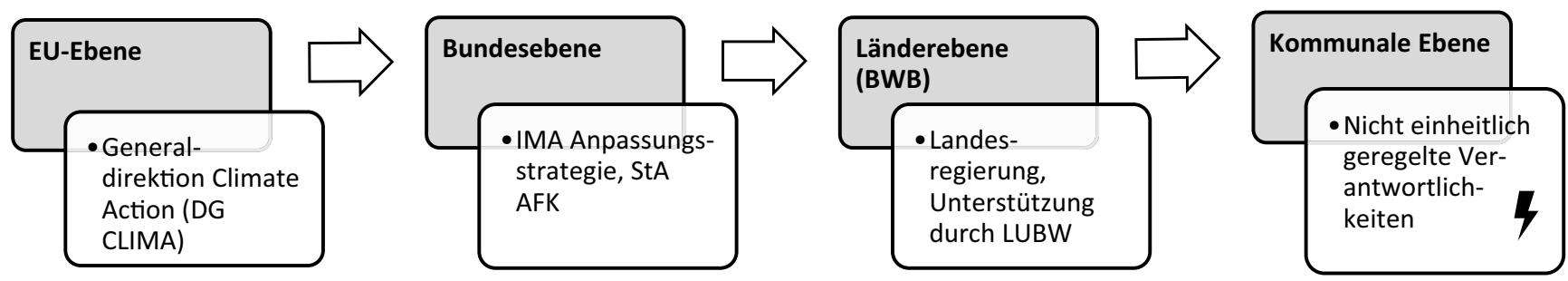

Abb. 3 Verwaltungsebenen und Verantwortlichkeiten für Klimaanpassung. (Quelle: eigene Darstellung) 
berg unterstützt seit 2011 modellhafte Vorhaben zur Anpassung an den Klimawandel. Das Klimopass-Projekt „Klimalotse“ ist ein speziell auf die Bedürfnisse kleiner und mittelgroßer Kommunen angepasstes Informationsangebot des Bundesumweltministeriums (Gebhardt et al. 2017, S. 151). In 5 thematisch aufeinander aufbauenden Modulen (dargestellt über einen eigenen Webauftritt) werden kommunale Entscheider in ihrer Auseinandersetzung mit Klimafolgen und Anpassung in zahlreichen Aspekten konzeptionell informiert und unterstützt.

\section{- KLIWA}

Infolge der Hochwasserrisikomanagement-Richtlinie wurde 2018 eine vorläufige Risikobewertung für Starkregenereignisse in Baden-Württemberg verfasst. Die Ergebnisse sind öffentlich abrufbar und stellen mögliche Risikoszenarien für betroffene Regionen transparent dar. Anhand detaillierter Karten lassen sich die Verläufe übertretender Flüsse darstellen und entsprechende Maßnahmen auf lokaler Ebene ableiten. Im süddeutschen Kooperationsvorhaben KLIWA (Klimaveränderung und Konsequenzen für die Wasserwirtschaft) werden Auswirkungen des Klimawandels für gefährdete Regionen prognostiziert und Handlungsempfehlungen im Sinne nachhaltiger Wasserwirtschaft entwickelt (Worreschk 2014, S. 310).

Darüber hinaus wird mit dem IEKK Baden-Württemberg ein Handlungs- und Entscheidungsrahmen für Kommunen geschaffen, um konkrete Maßnahmen und Strategien langfristig in unterschiedliche Sektoren (Strom, Wärme, Verkehr, Landnutzung und Stoffströme) aufzunehmen (Flaskuehler 2018, S. 260f.). Die Umsetzung in der Praxis wurde seit 2014 in zahlreichen Gemeinden und Kommunen in Baden-Württemberg initiiert. Praxisbeispiele für Klimaschutzkonzepte, welche unter wissenschaftlicher Begleitung entstanden, finden sich für die Stadt Schwäbisch Gmünd, die Gemeinde Rainau (Ostalbkreis) und für den Landkreis Heidenheim.

\section{Bedeutung der präsentierten Rahmenbedingungen für Kommunen in Baden-Württemberg}

Die Ausarbeitung von Konzepten zur Klimaanpassung wird durch unterschiedliche Instrumente bereits politisch forciert. Die gesetzliche Verbindlichkeit klimaanpassungsrelevanter Festlegungen und Richtlinien ist auf Bundes- und auf Landesebene jedoch noch nicht hinreichend gegeben (Schmitt 2016). Vor allem kleinen und mittelgroßen Kommunen fehlen zudem die nötigen finanziellen und strukturellen Mittel, um sich mit der Thematik der Klimaanpassung auseinanderzusetzen (Schinarakis 2016). Oftmals blei- ben die Verantwortlichkeiten auf kommunaler Ebene undefiniert. Die Forderung nach einer Integration von Anpassung in die Kommunalplanung wird zwar von unterschiedlichen Stellen formuliert, jedoch ohne die Benennung möglicher Zuständigkeiten (Knapp und Klotz 2017, S. 232).

Daraus resultierend stellt sich die Frage, wie die Integration von Anpassung in die Kommunalplanung zukünftig gelingen kann. Zur Diskussion stehen

- eine stärkere gesetzliche Bindung zur kommunalen Anpassung (Bubecka et al. 2016),

- vermehrte, monetäre Anreize durch Fördermaßnahmen (Hansjürgens et al. 2017, S. 255),

- die Möglichkeit zur Partizipation und Kooperation durch Einbindung Dritter (Vetter et al. 2017, S. 331)

oder eine Kombination von unterschiedlichen Instrumenten (Gaus et al. 2019, S. $94 \mathrm{ff}$.).

Als besonders aussichtsreich werden von zahlreichen Autoren sektorübergreifende Koordination und Abstimmungen angesehen (Roggero und Thiel 2015). Eine intensivierte Zusammenarbeit mit verschiedenen Stakeholdern der Region wäre an dieser Stelle denkbar. Baden-Württemberg verfügt über eine vergleichsweise hohe Hochschuldichte. Strategische Hochschulkooperationen könnten dazu beitragen, die mittelgroßen und kleineren Kommunen bei der Ideenfindung, Konzeption und Antragstellung für Fördermaßnahmen zu unterstützen. Auch gemeinsame, wissenschaftlich begleitete (Transfer-)Projekte für Strategien zum Umgang mit den spezifischen, regionalen Vulnerabilitäten könnten verstärkt initiiert werden.

Zudem sollten die Überlegungen der Klimaanpassung nicht an Ländergrenzen stoßen. Die Bildung geografischer Interessenscluster wäre ein möglicher Ansatz. Für BadenWürttemberg könnte beispielsweise ein potenzielles Interessenscluster im Zusammenschluss mit den alpennahen Gebieten Bayerns sowie mit den angrenzenden Alb-Regionen der Nachbarländer bestehen. Eine Einordnung anhand der Naturräume und Raumordnungsregionen (Diller und Hebecker 2008) wäre eine Option.

Informationen, Erfahrungswerte und bereits initiierte Maßnahmen und Forschungsvorhaben der geografisch assimilierten Regionen könnten besser vernetzt werden und zu sogenannten Win-win-Situationen führen. Die strategische Zusammenarbeit mit regionalen Stakeholdern könnte kommunalen Entscheidern helfen, die Empfehlungen der Bundes- und Landesprogramme effektiv in ihre Planungen zu integrieren.

Funding Open Access funding provided by Projekt DEAL.

Open Access Dieser Artikel wird unter der Creative Commons Namensnennung 4.0 International Lizenz veröffentlicht, welche die Nutzung, Vervielfältigung, Bearbeitung, Verbreitung und Wiedergabe in jeglichem Medium und Format erlaubt, sofern Sie den/die ursprüng- 
lichen Autor(en) und die Quelle ordnungsgemäß nennen, einen Link zur Creative Commons Lizenz beifügen und angeben, ob Änderungen vorgenommen wurden.

Die in diesem Artikel enthaltenen Bilder und sonstiges Drittmaterial unterliegen ebenfalls der genannten Creative Commons Lizenz, sofern sich aus der Abbildungslegende nichts anderes ergibt. Sofern das betreffende Material nicht unter der genannten Creative Commons Lizenz steht und die betreffende Handlung nicht nach gesetzlichen Vorschriften erlaubt ist, ist für die oben aufgeführten Weiterverwendungen des Materials die Einwilligung des jeweiligen Rechteinhabers einzuholen.

Weitere Details zur Lizenz entnehmen Sie bitte der Lizenzinformation auf http://creativecommons.org/licenses/by/4.0/deed.de.

\section{Literatur}

Beckmann S, Hiete M (2020) Predictors Associated with Health-Related Heat Risk Perception of Urban Citizens in Germany. Int J Environ Res Public Health 17(3):874. https://doi.org/10.3390/ ijerph17030874

Bubecka P, Klimmer L, Albrecht J (2016) Klimaanpassung in der rechtlichen Rahmensetzung des Bundes und Auswirkungen auf die Praxis im Raumordnungs-, Städtebau- und Wasserrecht. Natur Recht 38:297-307. https://doi.org/10.1007/s10357-016-3003-1

Deutschländer T, Mächel H (2017) Klimawandel in Deutschland: regionale Besonderheiten und Extreme. In: Brasseur G, Jacob D, Schuck-Zoeller S (Hrsg) Klimawandel in Deutschland: Entwicklung, Folgen, Risiken und Perspektiven. Springer Spektrum, Heidelberg, S 47-53

Diller C, Hebecker J (2008) Klimawandel in Deutschland: Handlungsmöglichkeiten der Regionalplanung. Standort Z Angew Geogr 32:62-70. https://doi.org/10.1007/s00548-008-0085-x

DWD (2019) Deutschlandwetter im Sommer 2019 Sonnenscheinreich und niederschlagsarm - neue Temperaturrekorde. Resource document. Deutscher Wetterdienst. https://www.dwd.de/DE/presse/ pressemitteilungen/DE/2019/20190830_deutschlandwetter_ sommer2019.pdf?_blob=publicationFile\&v=2. Zugegriffen: 26. Mai 2020

Fath K, Schmidt H, Stengel J, Wiens M, Schultmann F (2014) Analyse der industriellen Vulnerabilität gegenüber klimawandelinduzierten Risiken in Ballungsräumen Baden-Württembergs. Resource document. LUBW. http://fachdokumente.lubw. baden-wuerttemberg.de/servlet/is/117507/U83-W03-N18.pdf? command $=$ downloadContent \&filename $=$ U83-W03-N18.pdf \& FIS=91063. Zugegriffen: 16. Mai 2020

Flaskuehler C (2018) Föderale Klimaschutzgesetzgebung in Deutschland im Lichte des wohlgeordneten Rechts. Nomos, Baden-Baden

Gaus H, Silvestrini S, Kind C, Kaiser T (2019) Politikanalyse zur Evaluation der Deutschen Anpassungsstrategie an den Klimawandel (DAS) - Evaluationsbericht. Resource document. UBA. https:// www.umweltbundesamt.de/sites/default/files/medien/1410/ publikationen/politikanalyse_zur_evaluation_der_deutschen_ anpassungsstrategie_an_den_klimawandel_das_-evaluationsbericht.pdf. Zugegriffen: 23. Mai 2020

Gebhardt O, Meyer V, Meier F (2017) Leitfäden für die Anpassung an den Klimawandel - ein Überblick. In: Marx A (Hrsg) Klimaanpassung in Forschung und Politik. Springer Spektrum, Wiesbaden, S 143-182

Hansjürgens B, Schröter-Schlaak C, Berghöfer A, Bonn A, Dehnhardt A, Kantelhardt J, Liebersbach H et al (2017) Ökosystembasierte Klimapolitik für Deutschland. In: Marx A (Hrsg) Klimaanpassung in Forschung und Politik. Springer Spektrum, Wiesbaden, S 237-255
Haße C, Daschkeit A (2012) Zwischen Anpassung und Nachhaltigkeit - Aktuelle Klimaanpassung in Deutschland. Ökol Wirtsch 3:20-21. https://doi.org/10.14512/oew.v27i3.1215

Klimaks (2012) Klimaanpassungskonzept Stuttgart. Resource document. Landeshauptstadt Stuttgart. https://www.stadtklimastuttgart.de/stadtklima_filestorage/download/kliks/KLIMAKS2012.pdf. Zugegriffen: 23. Mai 2020

Knapp S, Klotz S (2017) Stadtnatur. In: Marx A (Hrsg) Klimaanpassung in Forschung und Politik. Springer Spektrum, Wiesbaden, S 215-232

Koerth J, Massmann F, Dittmann S (2019) Kommunale Klimaanpassung in Schleswig Holstein - Chancen und Herausforderungen. Standort Z Angew Geogr 43:177-184. https://doi.org/10.1007/ s00548-019-00602-4

LUBW (2019) Zu warm, zu heiß, zu trocken? Eine klimatische Einordnung des Jahres 2018 für Baden-Württemberg. Resource document. LUBW. https://pudi.lubw.de/detailseite/-/publication/ 83210. Zugegriffen: 7. Febr. 2020

LUBW (2020) Wieder außergewöhnlich warm und heiß, mit Nachwirkungen des Trockenjahrs 2018. Resource document. LUBW. https://pudi.lubw.de/detailseite/-/publication/10102. Zugegriffen: 16. Mai 2020

Marx A (2017) Klimawandel - ein Überblick. In: Marx A (Hrsg) Klimaanpassung in Forschung und Politik. Springer Spektrum, Wiesbaden, S 3-15

Obert M (2017) Anpassung an den Klimawandel als Aufgabe der Stadtplanung. In: Porth M, Schüttrumpf H (Hrsg) Wasser, Energie und Umwelt. Springer Vieweg, Wiesbaden, S 499-507

Ranke U (2019) Klima und Umweltpolitik. Springer, Berlin

Roggero M, Thiel A (2015) Unsicherheiten der Klimaforschung - Übergreifende Abstimmung in der Klimaanpassung. Ökol Wirtsch 1/2015:28-33. https://doi.org/10.14512/OEW300128

Schinarakis K (2016) Anpassung an den Klimawandel in Baden-Württemberg. Resource document. KIT. http://www.kit.edu/kit/pi_ 2016_069_anpassung-an-den-klimawandel-in-badenwuerttemberg.php. Zugegriffen: 11. Febr. 2020

Schindler D, Mayer H (2007) Forstmeterologische Untersuchungen zur Trockenheit 2003 im Südwesten Deutschlands. Allg Forst Jagd Z 178(2/3):21-37

Schmitt H (2016) Klimaanpassung in der Regionalplanung - Eine deutschlandweite Analyse zum Implementationsstand klimaanpassungsrelevanter Regionalplaninhalte. Raumforsch Raumordn 74:9-21. https://doi.org/10.1007/s13147-015-0375-2

UBA (2019) Der Weg zur deutschen Anpassungsstrategie - Anpassung auf Bundesebene. Resource document. UBA. https:// www.umweltbundesamt.de/themen/klima-energie/klimafolgenanpassung/anpassung-auf-bundesebene. Zugegriffen: 20. Mai 2020

UMBW (2014) Integriertes Energie- und Klimaschutzkonzept Baden-Württemberg. Resource document. UMBW. https://www. energieatlas-bw.de/documents/24384/24483/integriertes_Energie und_Klimaschutzkonzept_BW/237b8fd3-85d0-4444-9287f35124add081. Zugegriffen: 23. Mai 2020

UMBW (2015) Anpassungsstrategie des Landes Baden-Württemberg, Resource document. UMBW. https://um.baden-wuerttemberg.de/ fileadmin/redaktion/m-um/intern/Dateien/Dokumente/4_Klima/ Klimawandel/Anpassungsstrategie.pdf. Zugegriffen: 21. Mai 2020

UMBW (2019) Gesetzlicher Rahmen - Klimaschutzgesetz BadenWürttemberg. Resource document. UMBW. https://um.badenwuerttemberg.de/de/klima/klimaschutz-in-baden-wuerttemberg/ klimaschutzgesetz/. Zugegriffen: 13. Febr. 2020

Vetter A, Schauser I (2013) Anpassung an den Klimawandel: Priorisierung von Maßnahmen innerhalb der deutschen Anpassungsstrategie. Gaia Ökol Perspekt Wiss Ges 22(4):248-254 (https://search. proquest.com/docview/1477839209?accountid=132617)

Vetter A, Chrischilles E, Eisenack K, Kind C, Mahrenholz P, Pechan A (2017) Anpassung an den Klimawandel als neues Politikfeld. In: 
Brasseur G, Jacob D, Schuck-Zoeller S (Hrsg) Klimawandel in Deutschland: Entwicklung, Folgen, Risiken und Perspektiven. Springer, Heidelberg, S 325-334

Worreschk B (2014) Stand der Umsetzung der Hochwasserrisikomanagement-Richtlinie im Rheingebiet. In: Heimerl S, Meyer H (Hrsg)
Vorsorgender und nachsorgender Hochwasserschutz. Springer Vieweg, Wiesbaden, S 304-311 